\title{
Neurotoxic effects of domoic acid on dopaminergic neurons in primary mesencephalic cell culture
}

\author{
Khaled Radad ${ }^{1,2}$, Mubarak Al-Shraim ${ }^{1}$, Ahmed Al-Emam ${ }^{1,3}$, Rudolf Moldzio ${ }^{4}$, Wolf-Dieter Rausch ${ }^{4}$ \\ ${ }^{1}$ Department of Pathology, College of Medicine, King Khalid University, Abha, Saudi Arabia, ${ }^{2}$ Department of Pathology, \\ Faculty of Veterinary Medicine, Assiut University, Assiut, Egypt, ${ }^{3}$ Forensic and Toxicology Department, Faculty of Medicine, \\ Mansoura University, Mansoura, Egypt, ${ }^{4}$ Institute of Medical Biochemistry, Department for Biomedical Sciences, \\ University of Veterinary Medicine, Vienna, Austria
}

\begin{abstract}
Introduction: Domoic acid is a potent marine neurotoxin produced by certain species of the diatom genus Pseudonitzschia. To our knowledge, there are no studies that have investigated neurotoxic effects of domoic acid on dopaminergic neurons. Accordingly, the present study was carried out to investigate the potential neurotoxic effects of domoic acid on dopaminergic neurons in primary mesencephalic cell culture.

Material and methods: Cultures prepared from embryonic mouse mesencephala (total of 250 embryos) were treated with different concentrations of domoic acid $(0.1,1,10,100 \mu \mathrm{M})$ on the $10^{\text {th }}$ DIV for $48 \mathrm{~h}$. On the $12^{\text {th }}$ DIV, culture media were used for measurement of lactate dehydrogenase and cultured cells were subjected to immunostaining for tyrosine hydroxylase, neuronal nuclear antigen and glial fibrillary acidic protein, and fluorescence staining using $\mathrm{H}_{2} D C F D A, J C-1$ and DAPI stains. Moreover, roles of AMPA/KA and NMDA receptors in domoic acid neurotoxicity were also investigated.

Results: Domoic acid significantly decreased the number of dopaminergic neurons, decreased the expression of neuronal nuclear antigen and slightly affected astrocyte populations, and increased the release of lactate dehydrogenase into the culture media. AMPA/KA receptor antagonist NBQX but not NMDA receptor antagonist MK-801 significantly inhibited the neurotoxic effect of domoic acid on dopaminergic neurons. $H_{2} D C F D A, J C-1$ and DAPI fluorescence staining, respectively, revealed that DomA slightly raised ROS production, and significantly decreased mitochondrial membrane potential and increased apoptotic cell death of cultured cells.

Conclusion: The current study presents for the first time the neurotoxic effects of domoic acid on dopaminergic neurons and this effect appears to be attributed to activation of AMPA/KA receptors on dopaminergic neurons.
\end{abstract}

Key words: domoic acid, dopaminergic neurons, neurotoxicity, Parkinson's disease, marine, diatoms.

\section{Introduction}

Parkinson's disease (PD) is the second most prevalent neurodegenerative disease globally affecting about $1 \%$ of the population above 60 years. The disease is characterized neuropathologically by the loss of dopaminergic neurons in the substantia nigra pars compacta (SNpc) and formation of $\alpha$-synuclein-con-

\section{Communicating author}

Prof. Khaled Radad, Department of Pathology, College of Medicine, KKU University, Abha, Saudi Arabia, phone: +966 530984062 , e-mail: khaledradad@hotmail.com 
taining Lewy bodies [20]. The characteristic symptoms of PD are motor in nature, most notably tremor at rest, rigidity and bradykinesia. Non-motor symptoms like sleep problems, depression and loss of smell have recently gained an increased attention and were included among the diagnostic criteria [21]. Although PD has been extensively researched since its discovery by James Parkinson in 1817, the exact etiology and pathogenesis that underlie dopaminergic cell death are still unclear [11]. However, numerous epidemiological studies revealed that there is a strong association between sporadic PD which represents more than $90 \%$ of PD cases and environmental factors such as environmental toxins, pesticides and heavy metals [9]. Besides their colluding with the genetic agents, environmental factors have been demonstrated to cause nigrostriatal cell death through interfering with mitochondrial dysfunction, inducing oxidative stress and modifying proteasomal function [19].

Domoic acid (DomA) is a naturally occurring neurotoxin produced by some marine organisms such as the red alga Chondria armata and planktonic diatom of the genus Pseudo-nitzschia [6]. DomA can enter the food chain through accumulation in shellfish and finfish, and other types of seafood [2,8]. Consumption of these contaminated foods can lead to DomA intoxication in sea mammals, birds and humans [7]. In 1987, DomA caused an outbreak of human poisoning following consumption of DomA-contaminated blue mussels Mytilus edulis in eastern Canada [14], in which, four people died and more than 100 suffered from seizures, short-term memory loss, hallucinations and coma, a syndrome known as amnesic shellfish poisoning (ASP) $[14,16]$. The Center for Food Safety and Applied Nutrition (CFSAN), a branch of the Food and Drug Administration (FDA), has included ASP as one of the five recognized fish poisoning syndromes in the USA [12]. Neurotoxicity of DomA was reported in some in vitro and in vivo experimental models. For example, Giordano et al. [5] reported that exposure to acute intermediate-dose of DomA caused the significant apoptotic cell death in cerebellar granule neurons (CGNs). Vieira et al. [22] found that treatment of rats with DomA induced neuronal cell death, astrogliosis and microgliosis in the hippocampus, amygdale, olfactory tubercle, septal nuclei, piriform, perirhinal cortices and thalamus.

As so far no studies have shown the neurotoxic effects of DomA on dopaminergic neurons, the present study was conducted to investigate the neuro- toxic potential of DomA on dopaminergic neurons in primary mesencephalic cell culture relevant to PD.

\section{Material and methods \\ Preparation of primary mesencephalic cell cultures}

All experimental procedures in this study were done in accordance with the guidelines of the European Communities Directive of 24 November 1986 for the use of laboratory animals. In our experiments, primary mesencephalic cell cultures were prepared from the OF1/SPF embryos (250 embryos) at gestation day 14. After collecting the embryos under aseptic condition in Dulbecco's phosphate buffered saline (DPBS, Invitrogen, Germany), brains were released, mesencephala were excised and cultures were prepared according to Radad et al. [17]. Briefly, mesencephala were carefully cleaned from meninges, and enzymatically and mechanically dissociated using $0.2 \%$ trypsin solution (Invitrogen, Germany) and fire-polished Pasteur pipettes, respectively. Then, obtained cells were grown in Dulbecco's modified Eagle's medium (DMEM) supplemented with $10 \mathrm{mM}$ HEPES buffer, $4 \mathrm{mM}$ glutamine and $10 \%$ heat-inactivated fetal calf serum (FCS). The medium was exchanged with DMEM supplemented with FCS on the $1^{\text {st }}$ and $3^{\text {rd }}$ DIV. On the $5^{\text {th }}$ DIV, half of the medium was replaced with serum-free DMEM containing $0.02 \mathrm{ml} \mathrm{B-27/ml} \mathrm{(Invitrogen,} \mathrm{Germany).} \mathrm{Serum-}$ free DMEM supplemented with $0.02 \mathrm{ml} \mathrm{B}-27 / \mathrm{ml}$ was used for feeding of cultured cells from the $6^{\text {th }}$ DIV and was subsequently replaced every $2^{\text {nd }}$ day.

\section{Treatment of cultures with DomA}

A stock solution of $1 \mathrm{mM}$ DomA (Sigma, Germany) was prepared in distilled water and further diluted in DMEM to the final concentrations. On the $10^{\text {th }}$ DIV, cultures were treated with different concentrations of DomA $(0.1,1,10,100 \mu \mathrm{M})$ for $48 \mathrm{~h}$. The lethal concentration $\left(L C_{50}\right)$ that leads to loss of $50 \%$ of dopaminergic neurons was determined.

\section{Identification of dopaminergic neurons}

Dopaminergic neurons in primary mesencephalic cell cultures were identified by immunostaining against tyrosine hydroxylase $(\mathrm{TH})$. On the $12^{\text {th }}$ DIV, cultured cells were rinsed carefully with PBS (pH 7.2) and fixed with histochoice for $15 \mathrm{~min}$ at room temperature. Then, cultured cells were washed with PBS and permeabilized 
with $0.4 \%$ Triton X-100 for 30 min at room temperature. After washing 3 times with PBS, cultured cells were incubated with $5 \%$ horse serum (Vectastain ABC Elite kite) for $90 \mathrm{~min}$ to block non-specific binding sites. To visualize dopaminergic neurons (THir neurons), cultures were sequentially incubated with anti-TH primary antibody overnight at $4^{\circ} \mathrm{C}$, biotinylated secondary antibody (Vectastain) and avidin-biotin-horseradish peroxidase complex (Vectastain) for $90 \mathrm{~min}$ at room temperature. The reaction product was developed in a solution of diaminobenzidine $(1.4 \mathrm{mM})$ in PBS containing $3.3 \mathrm{mM}$ hydrogen peroxide $\left(\mathrm{H}_{2} \mathrm{O}_{2}\right)$. Dopaminergic neurons were counted with a Nikon inverted microscope in 10 randomly selected fields per well at 10x magnification.

\section{Detection the effects of DomA on total neuronal cells and astrocytes}

Two sets of cultures were treated with DomA (10 and $100 \mu \mathrm{M}$ ) on the $10^{\text {th }}$ DIV for $48 \mathrm{~h}$. On the $12^{\text {th }}$ DIV, cultures were stained immunocytochemically using anti-neuronal nuclear antigen (anti-NeuN) and anti-glial fibrillary acidic protein (anti-GFAP) antibodies (Chemicon, USA) for visualizing postmitotic neuronal cell types and astrocytes, respectively. The same staining procedures were carried out as described for anti-TH immunostaining except that the anti-TH antibody was replaced with the anti-NeuN or anti-GFAP antibodies.

\section{Measurement of lactate dehydrogenase (LDH) in the culture medium}

$\mathrm{LDH}$ is usually used to quantitatively assess cell damage. After treatment of cultures with different concentrations of DomA $(0.1,1,10,100 \mu \mathrm{M})$ on the $10^{\text {th }}$ DIV for $48 \mathrm{~h}$, culture media were collected and used to measure LDH with the cytotoxic detection kit according to the manufacturer's instructions. In brief, $\mathrm{NADH}+\mathrm{H}^{+}$produced from $\mathrm{NAD}^{+}$by $\mathrm{LDH}$ is transferred by diaphorase to the yellow tetrazolium salt 2-[4-iodophenyl]-3-[4-nitrophenyl]-5-phenyltetrazolium chloride (INT) resulting in red formazan formation. The latter was measured spectrophotometrically at $490 \mathrm{~nm}$ with a reference at $688 \mathrm{~nm}$. Supplemented medium was used as a blank and subtracted as background.

\section{Treatment of cultures with NBQX and MK-801}

To show the role of AMPA/KA and NMDA receptors in DomA neurotoxicity, two sets of cultures were separately pretreated with $10 \mu \mathrm{M}$ NBQX, an AMPA/ KA receptors antagonist and $10 \mu M$ MK-801, a NMDA receptor antagonist, respectively, on the $10^{\text {th }}$ DIV. After $10 \mathrm{~min}$, cultures were treated with DomA (10 and $100 \mu \mathrm{M}$ ) for $48 \mathrm{~h}$. On the $12^{\text {th }}$ DIV, cultures were stained using anti-TH antibody and the number of dopaminergic neurons was counted.

\section{Measurement of reactive oxygen species (ROS) with $\mathrm{H}_{2}$ DCFDA}

$\mathrm{H}_{2}$ DCFDA is a general oxidative stress indicator in cell cultures. A $50 \mathrm{mM}$ stock of $\mathrm{H}_{2}$ DCFDA in DMSO was diluted in colourless DMEM to a final concentration of $10 \mu \mathrm{M}$. Prepared cultures in black 96-well plates were treated with DomA (10 and $100 \mu \mathrm{M})$ on the $10^{\text {th }}$ DIV for $48 \mathrm{~h}$. On the $12^{\text {th }}$ DIV, culture media were removed and cells were incubated with $10 \mu \mathrm{M} \mathrm{H}_{2}$ DCFDA at $37^{\circ} \mathrm{C}$ for $30 \mathrm{~min}$. Cultured cells were then washed twice with colourless DMEM and kept with DPBS (100 $\mu \mathrm{l} /$ well). Semiquantitation of the fluorescence intensity was obtained by using a 2300 Enspire multilabel reader (Perkin Elmer) at an emission wavelength of $520 \mathrm{~nm}$. Data were transferred to Excel software and presented as means \pm SEM in which control was set to $100 \%$.

\section{Measurement of mitochondrial membrane potential $(\Delta \psi \mathrm{m})$ of cultured cells by JC- 1 fluorescence dye}

JC-1 is a cationic dye that can selectively accumulate in mitochondria by electrochemical gradient, indicated by a fluorescence emission shift from red $(\sim 590 \mathrm{~nm})$ to green $(\sim 529 \mathrm{~nm})$. At the end of the treatment incubation period, culture media were removed and cells were loaded with JC-1 (5 $\mu \mathrm{g} / \mathrm{ml}$ in DMEM) for $15 \mathrm{~min}$ at $37^{\circ} \mathrm{C}$. Mitochondrial depolarization is indicated by a decrease in the red/green fluorescence intensity ratio. Semiquantitation of fluorescence intensity was obtained by using black 96 well plates and a 2300 Enspire Multilabel Reader. Data were transferred to Excel software and presented as means \pm SEM in which control was set to $100 \%$.

\section{Counting of apoptotic cells by DAPI fluorescence dye}

As DAPI passes through intact cell membranes and binds to DNA, it is usually used to assess apoptotic nuclear changes. In which, cultures were treated with DomA (10 and $100 \mu \mathrm{M})$ on the $10^{\text {th }}$ DIV for $48 \mathrm{~h}$. After fixation of cultured cells with histochoice for $15 \mathrm{~min}$ at room temperature, cells were washed 


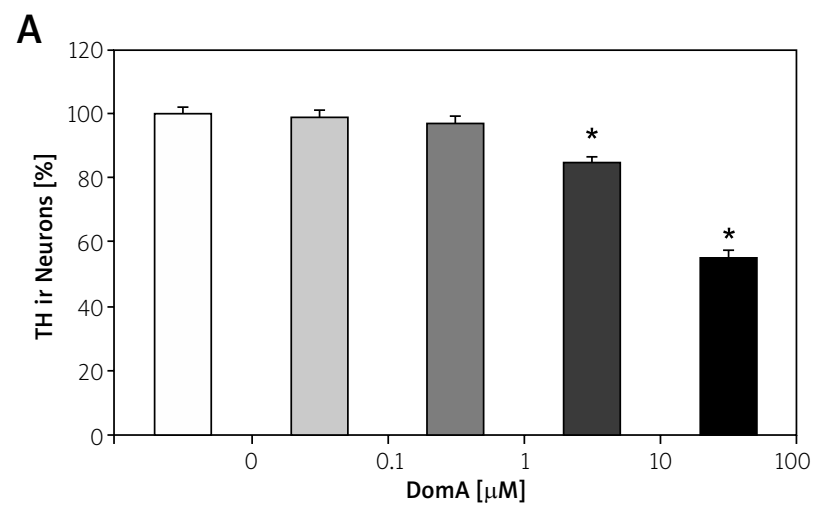

B
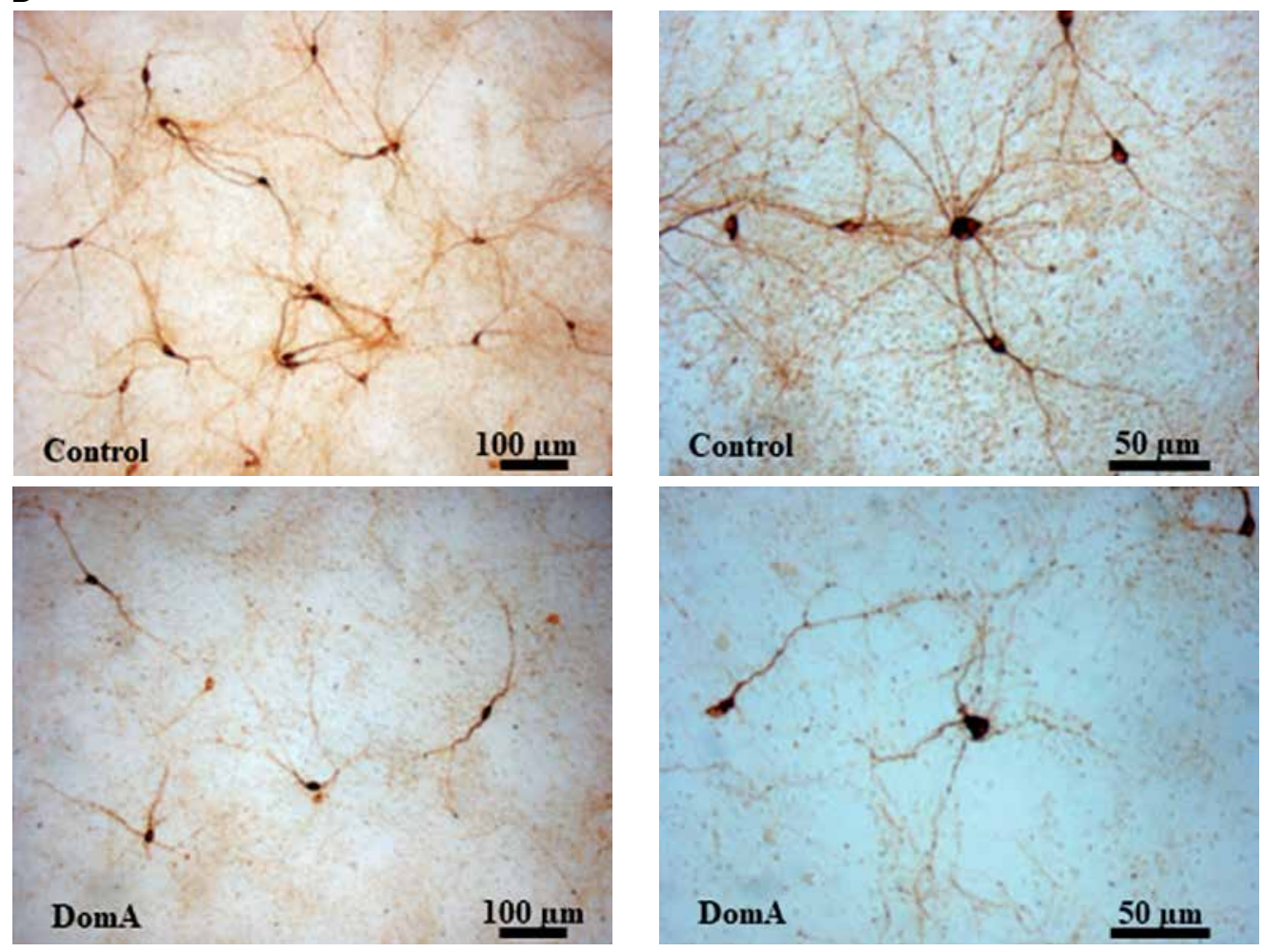

Fig. 1. Effects of DomA on the survival of dopaminergic neurons in primary mesencephalic cell culture. A) DomA (10 and $100 \mu \mathrm{M})$ significantly decreased the survival of dopaminergic neurons when added to the cultures from the $10-12^{\text {th }}$ DIV. $100 \%$ corresponds to the total number of dopaminergic neurons (the average number was 20.75 cells/field) after 12 DIV in untreated controls ( $\left.{ }^{*} p<0.001\right)$. B) Representative micrographs of dopaminergic neurons after 12 DIV. Control cultures showed many dopaminergic neurons with long and branched processes. DomA-treated cultures show few numbers of dopaminergic neurons with short and dysmorphic neurites.

with PBS (pH 7.2) and permeabilized with $0.4 \%$ Triton $\mathrm{X}-100$ for $30 \mathrm{~min}$ at room temperature. Then, cultured cells were loaded with $2 \mu \mathrm{M}$ DAPI solution for 5 min in the dark, washed with DPBS and photographed (6 photos/well) on an inverted fluorescence microscope using an ultraviolet (UV) filter (Nikon, Japan). Cells with condensed and fragmented chromatin were considered apoptotic.

\section{Statistics}

Three 4-well plates obtained from three different preparations were used to evaluate each experimental parameter. Data were expressed as mean \pm standard error of mean (SEM). Comparisons were made using ANOVA and post-hoc Duncan's test using IBM SPSS statistics $22 . P<0.05$ was considered as statistically significant. 

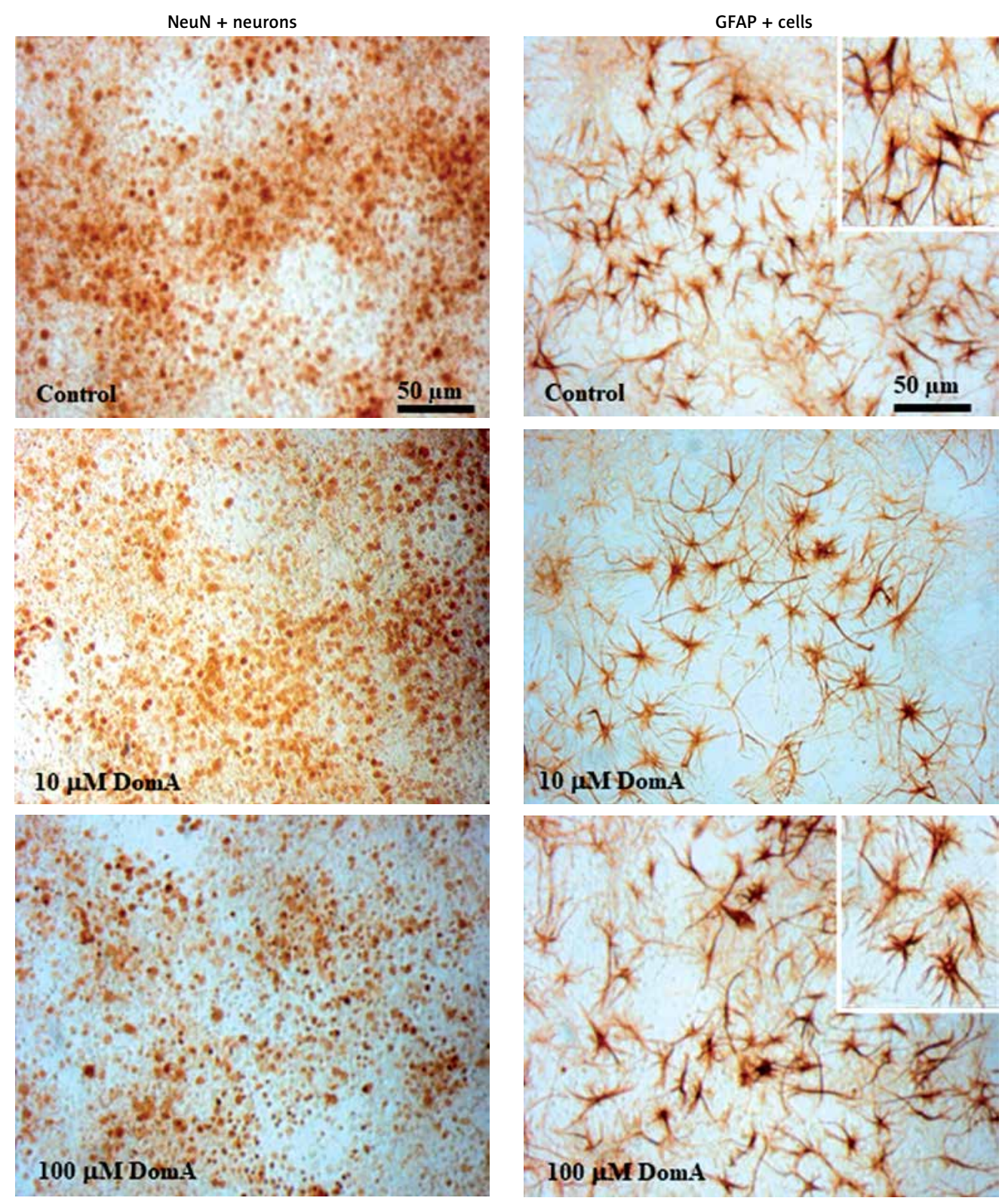

Fig. 2. Effects of DomA on the expression of neuronal and astrocyte markers NeuN and GFAP in primary mesencephalic cell culture. Treatment of cultures with DomA (10 and $100 \mu \mathrm{M})$ on the $10^{\text {th }}$ DIV for $48 \mathrm{~h}$ markedly decreased the expression of the neuronal marker NeuN and did not affect the expression of the astrocyte marker GFAP compared to untreated controls. Some astrocytes showed slight vacuolation compared to untreated controls (see the insets).

\section{Results}

\section{Neurotoxic effect of DomA on dopaminergic neurons}

Treatment of primary mesencephalic cell cultures with different concentrations of DomA $(0.1,1,10$, $100 \mu \mathrm{M}$ ) on the $10^{\text {th }}$ DIV for $48 \mathrm{~h}$ significantly reduced the number of dopaminergic neurons by about 15 and $46 \%$ at the concentrations 10 and $100 \mu \mathrm{M}$, respectively, compared to untreated controls (Fig. 1A). The $\mathrm{LC}_{50}$ of DomA for dopaminergic neurons was 100 MM (Fig. 1A). Moreover, DomA altered the morphology of surviving dopaminergic neurons. It decreased the number and length of their neurites compared 
to untreated cells which showed long and branched neurites (Fig. 1B).

\section{Effect of DomA on the total neuronal cells and astrocytes}

Incubation of primary mesencephalic cell cultures with 10 and $100 \mu \mathrm{M}$ DomA on the $10^{\text {th }}$ DIV for $48 \mathrm{~h}$ decreased the expression of the neuronal marker NeuN compared to untreated controls (Fig. 2). On the other hand, similar treatment of cultures with DomA did not affect the expression of the astrocyte marker GFAP. However, some astrocytes showed slight shrinkage and vacuolation at the concentration $100 \mu \mathrm{M}$ compared to untreated controls (Fig. 2, see the insets).

\section{Effect of DomA acid on LDH release}

Consistent with its neurotoxic effect on dopaminergic and NeuN+ neurons, DomA showed an overall toxic effect on primary mesencephalic cell culture as it increased LDH release into the culture medium by $83 \%$ at the concentration $100 \mu \mathrm{M}$ compared to untreated controls (Fig. 3).

\section{Effects of NBQX and MK-801 on DomA-induced neurotoxicity $\mathrm{n}$ dopaminergic neurons}

When primary mesencephalic cell cultures were separately treated with NBQX and MK-801, the AMPA/

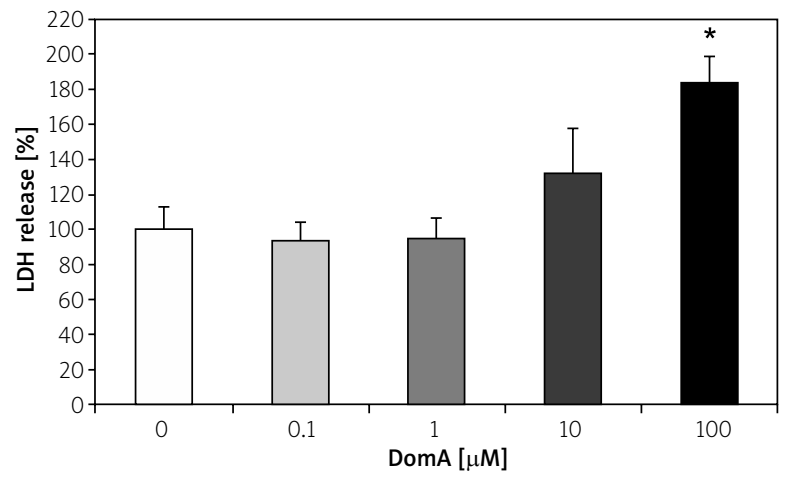

Fig. 3. Effects of DomA on the release of LDH into the culture medium. DomA $(100 \mu \mathrm{M})$ significantly increased LDH release into the culture medium when added from the $10-12^{\text {th }}$ DIV. $100 \%$ corresponds to the amount of LDH in the culture medium after 12 DIV $\left({ }^{*} p<0.01\right)$.
KA and NMDA receptor antagonists, 10 min prior to DomA, a significant number of dopaminergic neurons (23\%) was rescued in the cultures treated with NBQX but not MK-801 compared to DomA-treated cultures (Fig. 4A). Fig. 3B shows a higher number of dopaminergic neurons in cultures co-treated with NBQX and DomA compared to those treated with DomA alone. On the other hand, no difference between cultures co-treated with MK-801 and DomA, and those treated with DomA alone was observed (Fig. 4B).

\section{Effect of DomA on ROS production in cultured cells}

DomA at concentrations 10 and $100 \mu \mathrm{M}$ increased ROS production by $12-23 \%$, respectively, which did not reach statistical significance compared to untreated control cultures (Fig. 5A).

\section{Effect of DomA on $\Delta \psi \mathrm{m}$ of cultured cells}

Incubation of cultures with DomA on the $10^{\text {th }}$ DIV for $48 \mathrm{~h}$ significantly reduced the red/green fluorescence ratio of JC- 1 by $20 \%$ and $27 \%$ at the concentrations 10 and $100 \mu \mathrm{M}$, respectively, compared to untreated controls (Fig. 5B).

\section{Effect of DomA on apoptotic cell death of cultured cells}

DAPI staining showed that treatment of primary mesencephalic cell cultures with DomA on the $10^{\text {th }}$ DIV for $48 \mathrm{~h}$ significantly increased the number of apoptotic nuclei by $64 \%$ and $200 \%$ at the concentrations of 10 and $100 \mu \mathrm{M}$, respectively, compared to untreated controls (Fig. 6A). Apoptotic nuclei showed shrinkage, fragmentation and chromatin condensation compared to untreated controls (Fig. 6B).

\section{Discussion}

As currently there is a broad range of environmental factors linked to neurodegeneration, scientific understanding of the role of these environmental agents in the development of neurodegenerative diseases is critical and will help develop new therapies. Accordingly, the present study adopted primary mesencephalic cell culture to test the potential neurotoxic effect of DomA and reveal whether it could be implicated in PD. DomA at higher concentrations $(10,100 \mu \mathrm{M})$ was shown to significantly decrease the number and alter the morphology of dopaminer- 
A

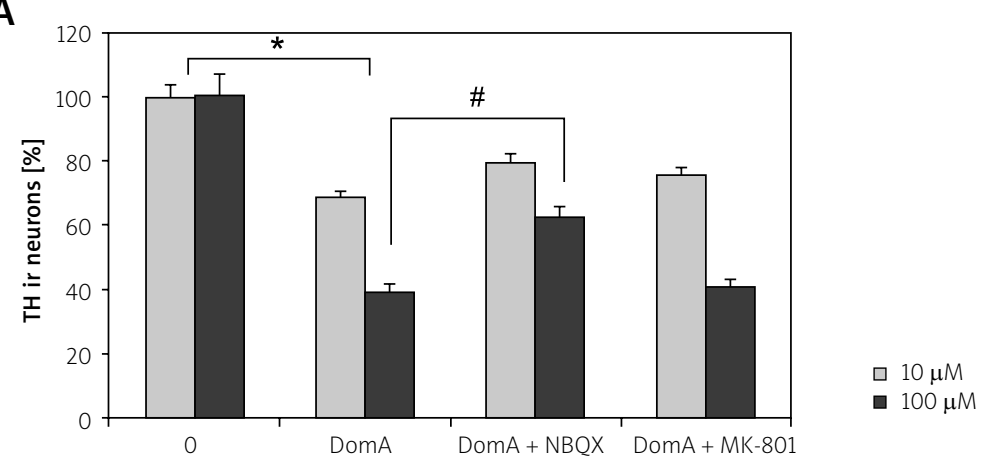

B
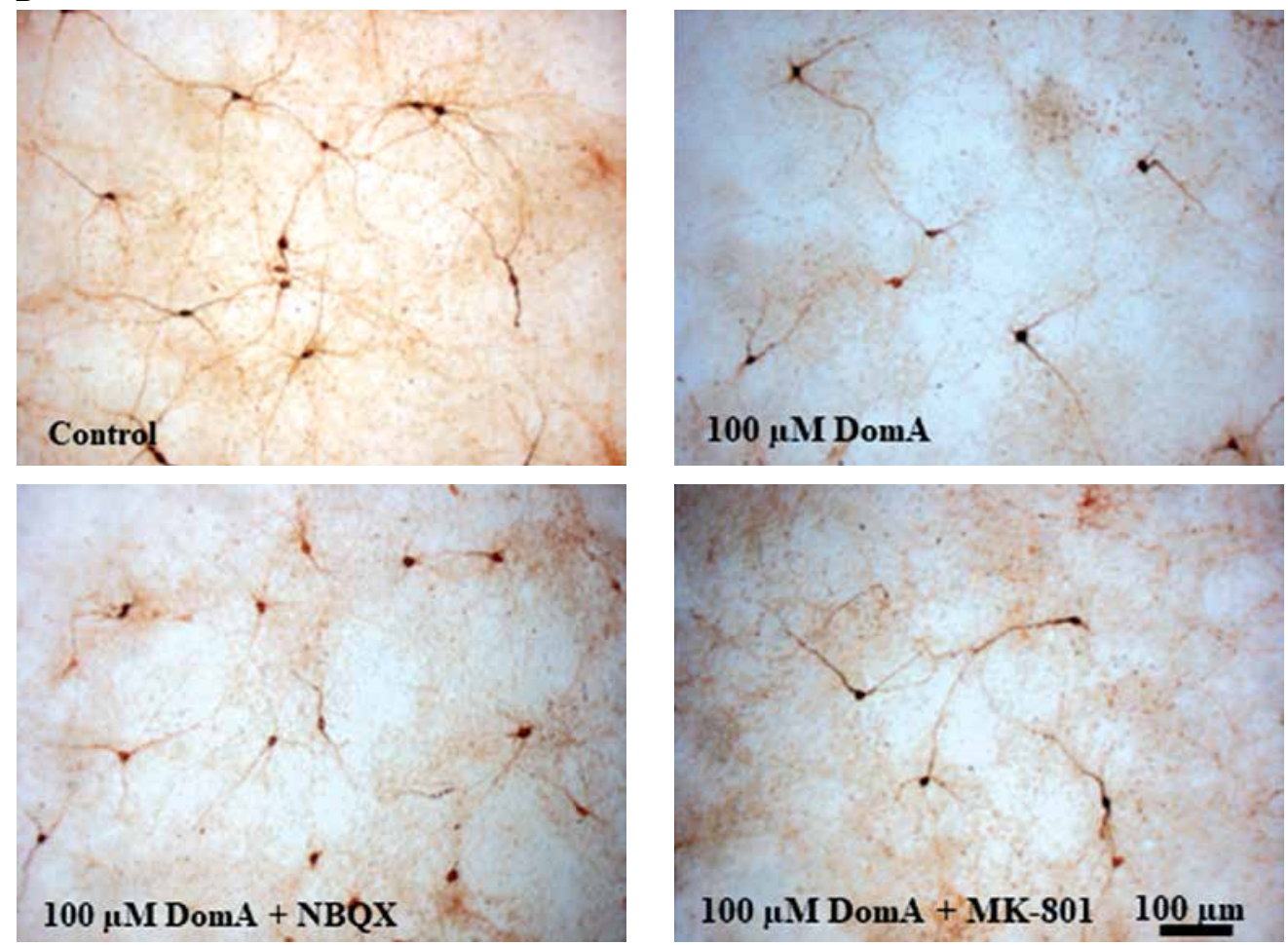

Fig. 4. Effects of NBQX and MK-801 on DomA neurotoxicity in primary mesencephalic cell culture. A) DomA $(10$ and $100 \mu \mathrm{M})$ significantly reduced the number of dopaminergic neurons when added to the cultures from the $10-12^{\text {th }}$ DIV compared to untreated controls $\left({ }^{*} p<0.001\right)$. NBQX and not MK-801 rescued a significant number of dopaminergic neurons in the $100 \mu \mathrm{M}$ DomA-treated cultures ( $\left.{ }^{\prime} p<0.001\right) .100 \%$ corresponds to the total number of dopaminergic neurons after 12 DIV in untreated controls. B) Representative micrographs of dopaminergic neurons after 12 DIV. NBQX- and not MK-801-co-treated cultures showed a higher number of dopaminergic neurons compared to cultures treated with DomA alone.

gic neurons in primary mesencephalic cell culture. In addition, it markedly decreased the expression of NeuN and slightly affected astrocyte populations. Similar neurotoxic effects of DomA were reported in some different in vitro and in vivo animal models since the 1987's outbreak in Eastern Canada. For instance, Xu et al. [24] and Giordano et al. [5] reported that exposure to DomA induced cell death in the motor neuron-like cells (NSC34) and mouse cerebellar granule cells (CGN), respectively. In in vivo animal models, Costa et al. [2] found that oral administration of DomA to rats caused some behavioral abnormalities such as hind limb scratching followed by seizures and hippocampal degeneration. Vieira et al. [22] reported that treatment of rats with DomA induced neuronal cell death in hippocampus 

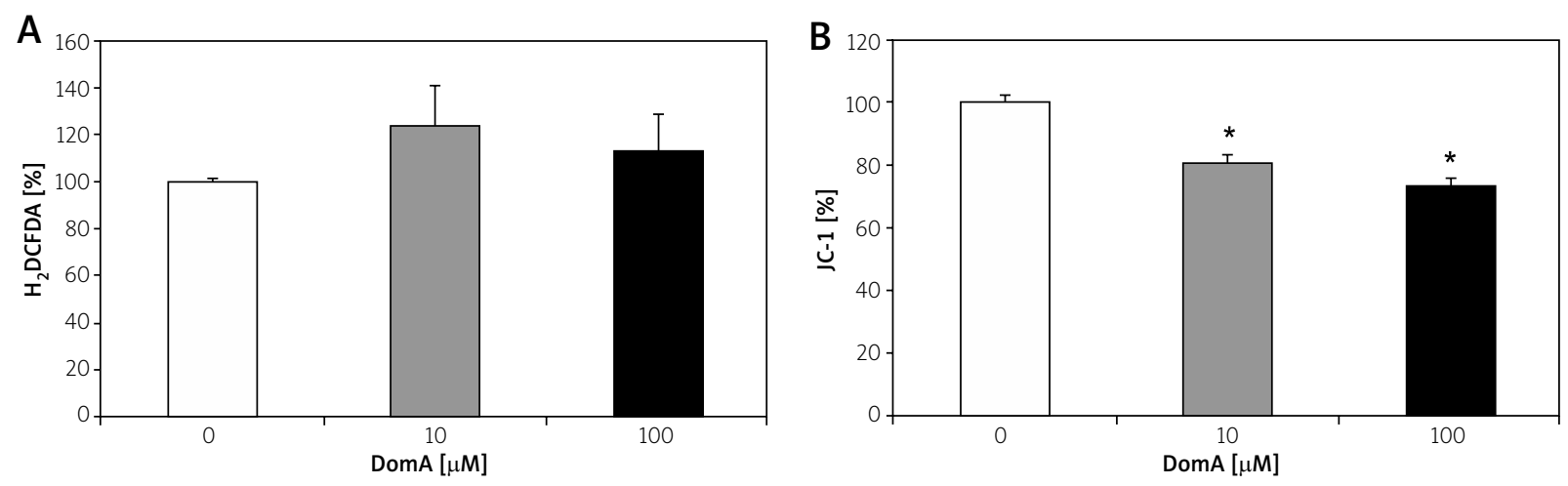

Fig. 5. Effects of DomA on ROS production and $\Delta \psi m$ in primary mesencephalic cell culture. A) Treatment of cultures with DomA $(10$ and $100 \mu \mathrm{M})$ on the $10^{\text {th }}$ DIV for $48 \mathrm{~h}$ resulted in an insignificant increase in ROS compared to untreated controls. B) Treatment of cultures with DomA (10 and $100 \mu \mathrm{M})$ on the $10^{\text {th }}$ DIV for $48 \mathrm{~h}$ significantly decreased the red:greed fluorescence ratio of JC-1 in cultured cells compared to untreated controls. $100 \%$ corresponds to the level of ROS and $\Delta \psi \mathrm{m}$ in untreated control cultures after 12 DIV $\left({ }^{*} p<0.01\right)$.

A

B
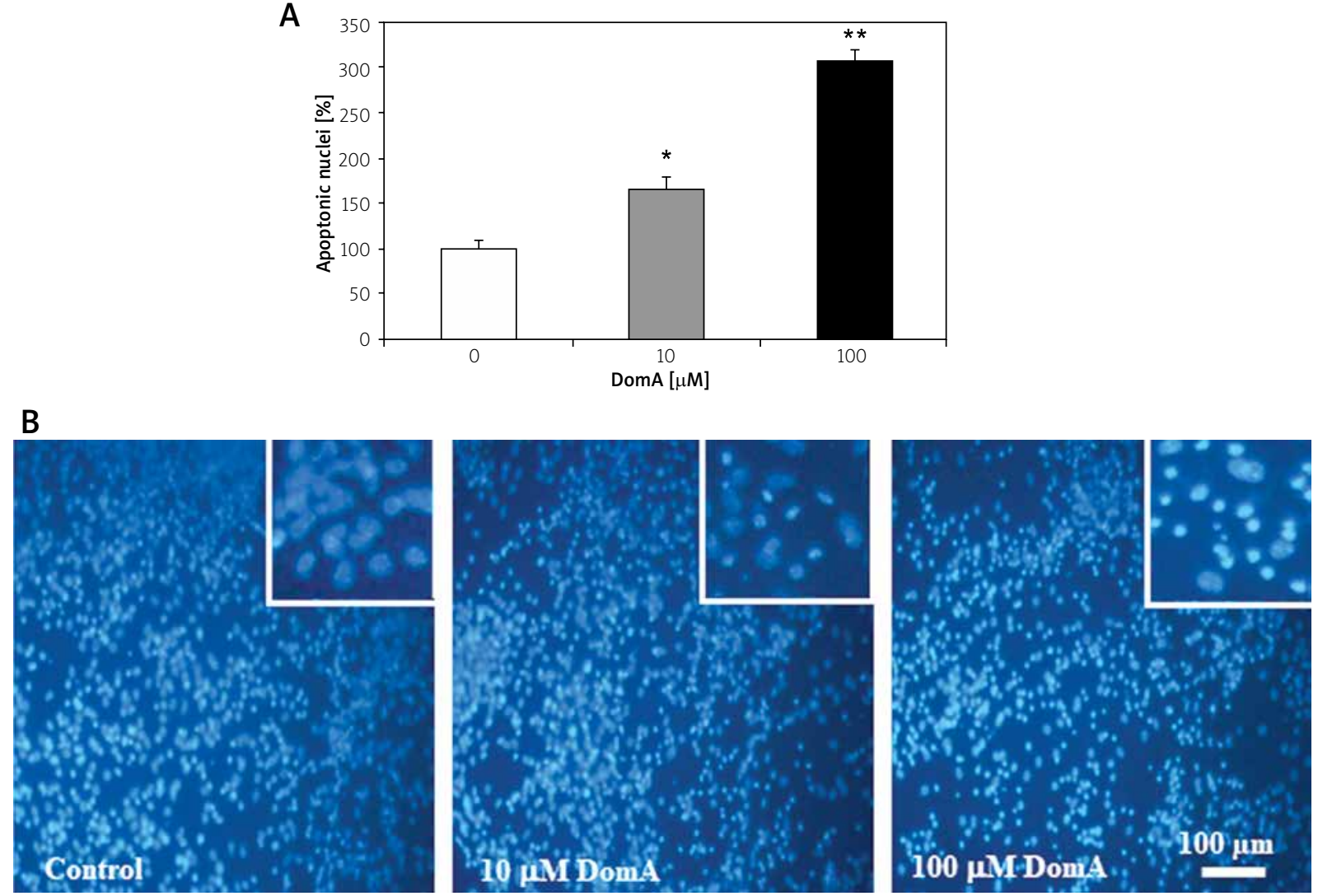

Fig. 6. Effects of DomA on apoptotic cell death in primary mesencephalic cell culture. A) Treatment of cultures with DomA (10 and $100 \mu \mathrm{M})$ on the $10^{\text {th }}$ DIV for $48 \mathrm{~h}$ significantly increased the numbers of apoptotic nuclei showing shrinkage, fragmentation and chromatin condensation. 100\% corresponds to the number of apoptotic nuclei (the average number was 185 nuclei/photo) in untreated control cultures after 12 DIV $\left({ }^{*} p<0.01,{ }^{* *} p<0.0001\right)$. B) Representative micrographs show increased numbers of apoptotic nuclei in DomA-treated cultures compared to untreated controls. Insets show normal and apoptotic nuclei at 20x magnification. 
and amygdala. Consistent with the slight effects on astrocytes, Gill et al. [3] found that DomA had no effect on GFAP expression in the cultures of primary astrocytes prepared from the hippocampus and brain stem. Moreover, DomA significantly increased $\mathrm{LDH}$ release into the culture medium at the concentration of $100 \mu \mathrm{M}$. Likewise, release of LDH into the culture medium was reported by Berman and Murray [1] following treatment of cerebellar granule neurons by DomA. A higher LDH concentration in the culture medium usually serves as a marker of general cytotoxicity and reflects a necrotic cell death. Both increase of LDH in the culture medium and decrease of NeuN expression reveal that DomA neurotoxicity was not restricted or selective to dopaminergic neurons in primary mesencephalic cell culture which form $1-2 \%$ of culture neuronal populations.

DomA as a structural analog of kainic acid was reported to induce its neurotoxicity through activation of AMPA/KA receptors. In this context, Larm et al. [10] reported that DomA produced neuronal cell death in primary cortical neurons via stimulating AMPA and KA-sensitive glutamate receptors. Giordano et al. [4] found that DomA induced apoptotic cell death in mouse cerebellar granule cells through activation of AMPA/KA receptors. Pérez-Gómez et al. [13] found that the neurotoxic effect of DomA on primary cultured cerebellar granule neurons was fully prevented by the AMPA/KA antagonist. Similarly in the present study, the neurotoxic effect of DomA on dopaminergic neurons appeared to be mediated by the activation of AMPA/KA receptor as treatment of cultures with the AMPA/KA receptor antagonist NBQX prior to DomA administration rescued a significant number of dopaminergic neurons compared to the cultures treated with DomA alone. Activation of AMPA/KA by higher concentrations of DomA in some in vitro cellular models was reported to provoke an increase of intracellular calcium $\left(\mathrm{Ca}^{2+}\right)$ levels leading to release of the glutamate neurotransmitter which in turn activates NMDA receptors [2]. Activation of NMDA receptors is further resulting in increased intracellular $\mathrm{Ca}^{2+}$ concentrations leading to an oxidative stress (OS)-induced mitochondrial dysfunction and activation of caspases, events leading to apoptotic cell death [5]. Inconsistently, NMDA receptors were seen to have no role in DomA-induced neurotoxicity on dopaminergic neurons in our culture model. This is because pretreatment of cultures with the NMDA receptor antagonist MK-801 did not block DomA neurotoxicity on dopaminergic neurons. In parallel, Pérez-Gómez et al. [13] reported that NMDA receptor was not involved in DomA-induced neurotoxicity in primary cultured cerebellar granule neurons.

Fluorescence staining of cultured cells with $\mathrm{H}_{2}$ DCFAD, JC-1 and DAPI stains respectively revealed that DomA increased production of ROS which did not reach significance, decreased the red : green fluorescence ratio of JC-1 and increased the number of apoptotic nuclei compared to untreated control cultures. Similarly, it was reported that DomA neurotoxicity was mediated by oxidative insults in Caco-2 cells [18], mitochondrial dysfunction in rat's cardiac muscle [23] and DNA damage in Caco-2 cells [15]. Taken collectively, these findings provide important insights into the underlying mechanisms of DomA neurotoxicity on cultured dopaminergic cells. An insignificant increase in ROS production by the higher concentrations of DomA (10 and $100 \mu \mathrm{M})$ might indicate that activation of AMPA/KA increased $\mathrm{Ca}^{2+}$ to a level that is insufficient to release much glutamate but on the other hand, can damage mitochondria as evaluated by JC-1 fluorescence staining. Mitochondrial damage seemed to mediate both apoptotic and necrotic cell death of cultured cells. In contrast, apoptotic cell death was reported to occur at the lower concentrations of DomA $(<1 \mu \mathrm{M})$ in mouse CGNs [5]. These lower concentrations did not affect both dopaminergic and $\mathrm{NeuN}+$ neurons in our culture model.

In conclusion, our study presents for the first time the neurotoxic effects of the marine neurotoxin DomA on dopaminergic neurons in primary mesencephalic cell culture. This effect appears to be attributed to activation of AMPA/KA receptors on dopaminergic neurons.

\section{Acknowledgments}

The authors are thankful to the Deanship of Scientific Research at the King Khalid University for the financial support of project no. KKU-GRP-172-38.

\section{Disclosure}

The authors report no conflict of interest.

\section{References}

1. Berman FW, LePage KT, Murray TF. Domoic acid neurotoxicity in cultured cerebellar granule neurons is controlled preferentially by the NMDA receptor $\mathrm{Ca}(2+)$ influx pathway. Brain Res 2002; 924: 20-29. 
2. Costa LG, Giordano G, Faustman EM. Domoic acid as a developmental neurotoxin. Neurotoxicology 2010; 31: 409-423.

3. Gill SS, Hou Y, Ghane T, Pulido OM. Regional susceptibility to domoic acid in primary astrocyte cells cultured from the brain stem and hippocampus. Mar Drugs 2008; 6: 25-38.

4. Giordano G, White CC, Mohar I, Kavanagh TJ, Costa LG. Glu tathione levels modulate domoic acid induced apoptosis in mouse cerebellar granule cells. Toxicol Sci 2007; 100: 433-444.

5. Giordano G, Klintworth HM, Kavanagh TJ, Costa LG. Apoptosis induced by domoic acid in mouse cerebellar granule neurons involves activation of p38 and JNK MAP kinases. Neurochem Int 2008; 52: 1100-1105.

6. Hiolski EM, Ito S, Beggs JM, Lefebvre KA, Litke AM, Smith DR. Domoic acid disrupts the activity and connectivity of neuronal networks in organotypic brain slice cultures. Neurotoxicology 2016; 56: 215-224.

7. Jandová K, Kozler P, Langmeier M, Marešová D, Pokorný J, Riljak V. Influence of low-dose neonatal domoic acid on the spontaneous behavior of rats in early adulthood. Physiol Res 2014; 63: 521-528.

8. Jeffery B, Barlow T, Moizer K, Paul S, Boyle C. Amnesic shellfish poison. Food Chem Toxicol 2004; 42: 545-557.

9. Lan AP, Chen J, Chai ZF, Hu Y. The neurotoxicity of iron, copper and cobalt in Parkinson's disease through ROS-mediated mechanisms. Biometals 2016; 29: 665-678.

10. Larm JA, Beart PM, Cheung NS. Neurotoxin domoic acid pro duces cytotoxicity via kainate- and AMPA-sensitive receptors in cultured cortical neurones. Neurochem Int 1997; 31: 677-82.

11. Li S, Dong J, Cheng C, Le W. Therapies for Parkinson's disease: alternative to current pharmacological interventions. Neura Transm 2016; 123: 1279-1299.

12. Mayer AM, Hall M, Fay MJ, Lamar P, Pearson C, Prozialeck WC, Lehmann VK, Jacobson PB, Romanic AM, Uz T, Manev H. Effect of a short-term in vitro exposure to the marine toxin domoic acid on viability, tumor necrosis factor-alpha, matrix metalloproteinase-9 and superoxide anion release by rat neonata microglia. BMC Pharmacol 2001; 1: 7.

13. Pérez-Gómez A, Cabrera-García D, Warm D, Marini AM, Salas Puig J, Fernández-Sánchez MT, Novelli A. Selective Enhancement of Domoic Acid Toxicity in Primary Cultures of Cerebellar Granule Cells by Lowering Extracellular $\mathrm{Na}+$ Concentration. Toxicol Sci 2018; 161: 103-114.

14. Perl TM, Bédard L, Kosatsky T, Hockin JC, Todd EC, Remis RS. An outbreak of toxic encephalopathy caused by eating mus sels contaminated with domoic acid. N Engl J Med 1990; 322 1775-1780.

15. Pinto-Silva CR, Moukha S, Matias WG, Creppy EE. Domoic acid induces direct DNA damage and apoptosis in Caco-2 cells: recent advances. Environ Toxicol 2008; 23: 657-663.

16. Quilliam MA, Wright JL. The amnesic shellfish poisoning mystery. Anal Chem 1989; 61: 1053A-106A.

17. Radad K, Moldzio R, Rausch WD. Rapamycin protects dopaminergic neurons against rotenone-induced cell death in primary mesencephalic cell culture. Folia Neuropathol 2015; 53: 250 261.
18. Ramya EM, Kumar GP, Anand T, Anilakumar KR. Modulatory effects of Terminalia arjuna against domoic acid induced toxicity in Caco-2 cell line. Cytotechnology 2017; 69: 725-739.

19. Schapira AH. Etiology of Parkinson's disease. Neurology 2006; 66: 10-23.

20. Scott L, Dawson VL, Dawson TM. Trumping neurodegeneration: Targeting common pathways regulated by autosomal recessive Parkinson's disease genes. Exp Neurol 2017; 298: 191-201.

21. Tysnes OB, Storstein A. Epidemiology of Parkinson's disease. J Neural Transm 2017; 124: 901-905.

22. Vieira AC, Alemañ N, Cifuentes JM, Bermúdez R, Peña ML, Botana LM. Brain Pathology in Adult Rats Treated With Domoic Acid. Vet Pathol 2015; 52: 1077-1086.

23. Vieira AC, Cifuentes JM, Bermúdez R, Ferreiro SF, Castro AR, Botana LM. Heart Alterations after Domoic Acid Administration in Rats. Toxins (Basel) 2016; 8: 3.

24. Xu R, Tao Y, Wu C, Yi J, Yang Y, Yang R, Hong D. Domoic acid induced spinal cord lesions in adult mice: evidence for the possible molecular pathways of excitatory amino acids in spinal cord lesions. Neurotoxicology 2008; 29: 700-707. 